\title{
Rare Manifestation of Churg-Strauss Syndrome: Coronary Artery Vasospasm Complicated by Myocardial Infarction

\begin{abstract}
Radi Fatima Zohra ${ }^{1 *}$, Hara Loubna ${ }^{1 *}$, El ouazzani Amine1, Berroho Ouassima1, Doghmi Nawal ${ }^{1}$, Zarzur Jamila1, Cherti Mohamed', Lahma Jawad ${ }^{2}$, El Ayoubi Ali², Essakali Leila², Tazi Mzaalak Zoubida ${ }^{3}$, Sefiani Sanae ${ }^{4}$
\end{abstract}

\section{Abstract}

Churg-Strauss syndrome (CSS) is a rare multisystemic disorder of unknown origin and cardiac involvement is one of the most serious manifestations of the disease. Vasospastic angina and myocardial infarction are unusual clinical manifestations of Churg-Strauss syndrome (CSS).

We report a case of Churg-Strauss syndrome coexistent with myocardial infarction due to coronary vasospasm. A 25 -year-old man with bronchial asthma and allergic rhinitis was admitted to our hospital because of acute respiratory distress and recurrent chest pain. Angiography showed severe spontaneous coronary artery spasm with healthy arteries. Cardiac MRI objectify a transmural ischemic involvement. Myocardial infarction due to coronary vasospasm was diagnosed. In addition, marked eosinophilia, eosinophilic pneumonitis, chronic sinusitis, a nasal endoscopy was performed objectifying nasal polyposis. The histological analysis of the polyposis biopsy revealed extravascular eosinophilic infiltrates. The patient was put on a combination therapy of glucocorticoids and cyclophosphamide.

\section{Keywords}

Coronary Artery Vasospasm, Churg Strauss Syndrome, Myocardial Infarction.
1 Department of cardiology B, university hospital Ibn Sina, Rabat, Morocco.

2 Department of otolaryngology, university hospital Ibn Sina, Rabat, Morocco.

3 Department of internal medicine, university hospital Ibn Sina, Rabat, Morocco.

4 Department of anatomical pathology, university hospital Ibn Sina, Rabat, Morocco.

*: these authors contributed equally to this manuscript.

Contact information:

Hara Loubna.

झ loubna.hara@gmail.com

\section{Introduction}

Churg-Strauss syndrome (CSS) is a rare systemic disease characterized by necrotizing vasculitis and peripheral eosinophilia. Although the overall prognosis is good, clinical studies suggest that cardiac invol- 
vement is associated with an adverse prognosis [1]. The cardiac manifestations of CSS vary, including myocarditis, congestive heart failure and valvular disease, while coronary vasospasms are rare $[2,3]$. We herein report a new case of severe coronary vasospasm complicated by myocardial infarction related to Churg Strauss syndrome. The diagnosis of CSS was established based on the American College of Rheumatology Criteria for CSS [4]. This is the second case in which a CSS patient exhibited severe coronary artery spasms without a provocation test.

\section{Case report}

In January 2016, a 25 year-old male patient was referred to our institution from

another hospital with acute respiratory distress and recurrent chest pain. He also experienced general weakness and systemic symptoms such as malaise the weeks before.

His past medical history revealed no previous hospitalizations and no cardiovascular risk factors. Since 2008, the patient suffered from chronic sinusitis. In 2014, asthma was diagnosed, under anti obstructive therapy with beclomethasone.

At admission, physical examination was insignificant. An electrocardiogram showed ST segment elevation in inferior leads, T waves inversion in all derivations, and QS aspect in ASA and inferior (Figure 1).

Figure 1: T waves inversion in all derivations, and QS aspect in anteroseptoapical and inferior leads.

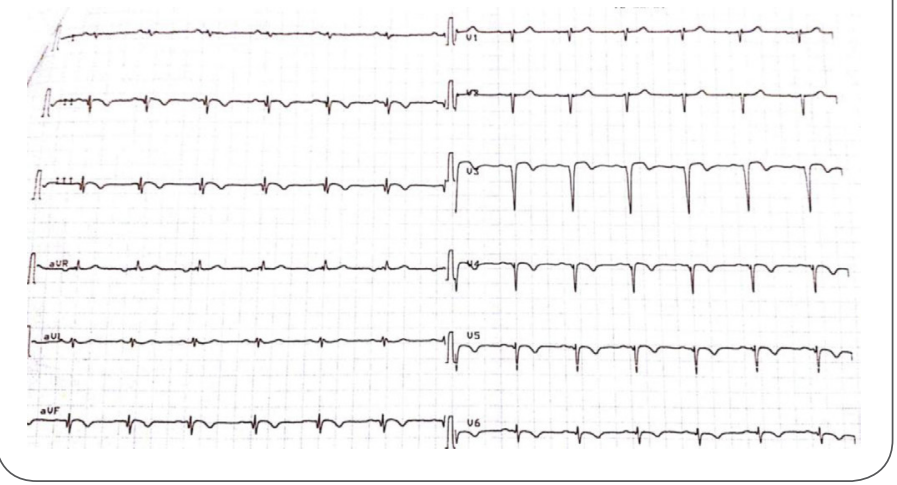

Figure 2: Reduced global longitudinal strain of the left ventricular.

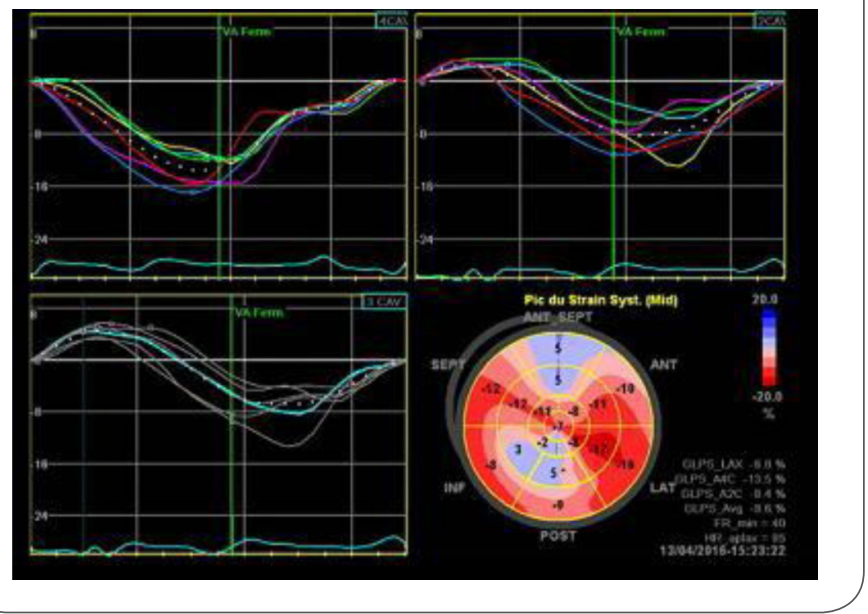

Initial laboratory findings revealed Troponine elevation $(0.88 \mu \mathrm{g} / \mathrm{l}$, reference value $<0.01 \mu \mathrm{g} / \mathrm{l})$, increased white blood cells $(12,050 / \mu \mathrm{l})$ and platelets $(326,000 / \mu \mathrm{l})$. In the course of diagnostic investigation differential WBC was performed and revealed eosinophilia of $20.2 \%$, corresponding with an abso-

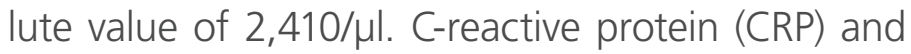
serum-creatinine as well as the glomerular filtration rate were in the normal range.

Serum test was negative for antinuclear antibody, rheumatoid factor, or antineutrophil cytoplasmic antibodies ( $p$-ANCA) measured using enzyme-linked immunosorbent assay.

Echocardiography demonstrated a severely damaged left ventricle with akinesia of the inferior well, the basal and the mid segment of the septum, the mid and apical segment of the inferolateral well, the basal of the anterior well, hypokinesia of the others wells, and alteration of global longitudinal strain $-9.5 \%$ (Figure 2).

Heart failure medication was introduced. As the patient had several asthma exacerbation after taking aspirin, this drug was withdrawn.

Coronary angiography revealed a spontaneous subtotal spastic occlusion of the mid of Left anterior descending artery (LAD) (Figure 3a) and the principal marginal (Figure $3 \mathbf{b}$ ) with total resolution of the spasm after intracoronary injection of nitroglyceri- 
Figure 3: Coronary angiography: a, b) Vasospastic stenosis of the mid of the left anterior interventricular artery (LAD) and the left marginal artery; $c, d$ ) their resolution after intracoronary nitroglycerine injection (e) Right coronary artery (RCA) angiography with no significant stenosis .

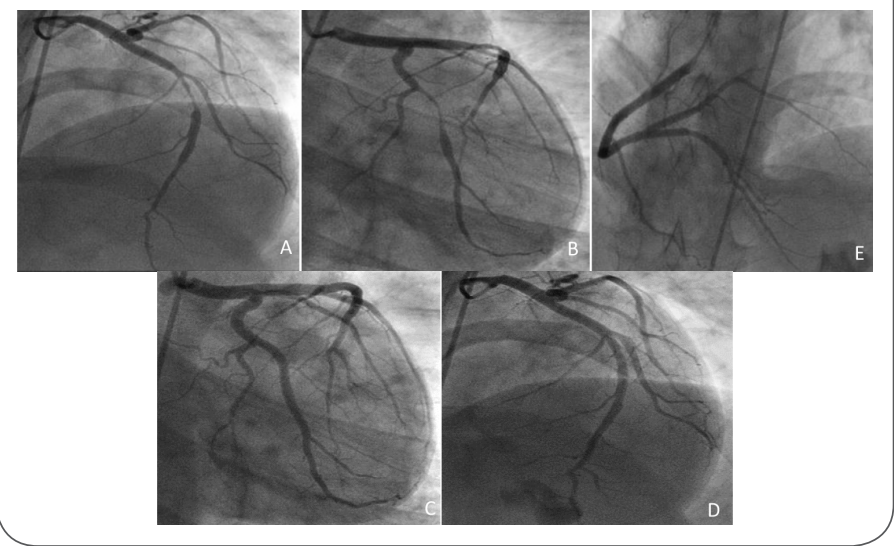

ne (Figure 3 c, d). Whereas, there was no critical stenosis in the right coronary artery angiography (Figure 3e).

Magnetic resonance imaging showed severe systolic dysfunction of left ventricular with $27 \%$ of ejection fraction and subendocardial delayed enchancement in the anterior, septal, inferolateral and inferior wells objectifying a transmural ischemic involvement (Figure 4).

View history of sinusite, a nasal endoscopy was performed objectifying nasal polyposis (Figure 5). The histological results of the polyposis biopsy revealed extravascular eosinophilic infiltrates (Figure $6)$.

The presence of asthma, hypereosinophilia $>10 \%$, chronic sinusitis, and eosinophilic proctitis represent 4 of the 6 ACR criteria required for the classification of CSS. In addition, cardiac involvement was present due to coronary vasospasm. Vasodilators did not relieve the patient's vasospastic angina; however, immunosuppressive therapy improved her symptoms, which lead us to initiate the combination therapy of glucocorticoids and cyclophosphamide with favourable clinical course.
Figure 4: Cardiac MRI: short axis view of left ventricular myocardium in delayed enchancement (DE) passed a) by basal; b) mid; $c, d)$ and apical segments showing left ventricular subendocardial delayd enchancement (blue arrow):

- in $50 \%$ of the myocardial thickness of the basal and mid segments of the anterior, anteroseptal, anterolateral and inferolateral wells,

- $\quad->70 \%$ of the myocardial thickness of the inferior, inferoseptal and all apical segment objectifying a transmural ischemic involvement of the left ventricular at this level.

To note in b) a delayed enchancement in the posterior pillar (red arrow).
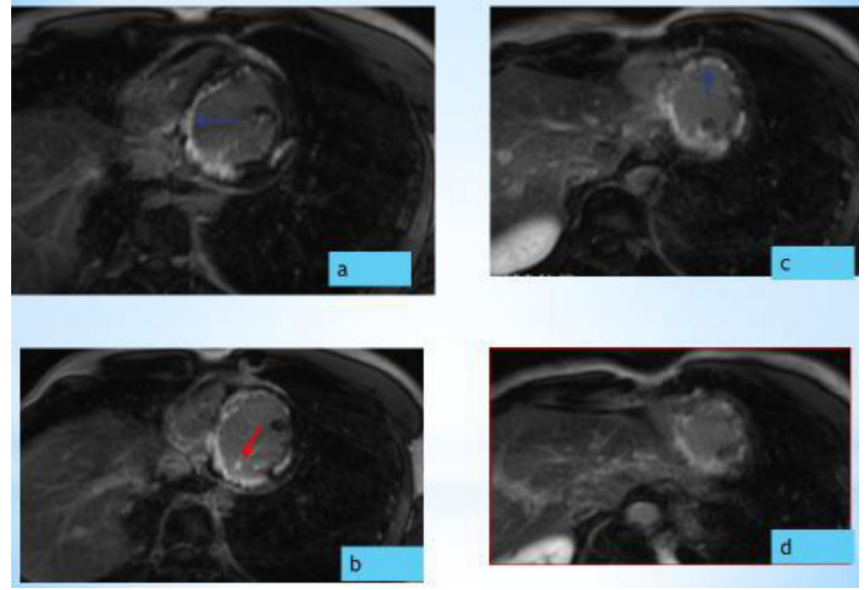

Figure 5: Nasal endoscopy showing nasal polyposis

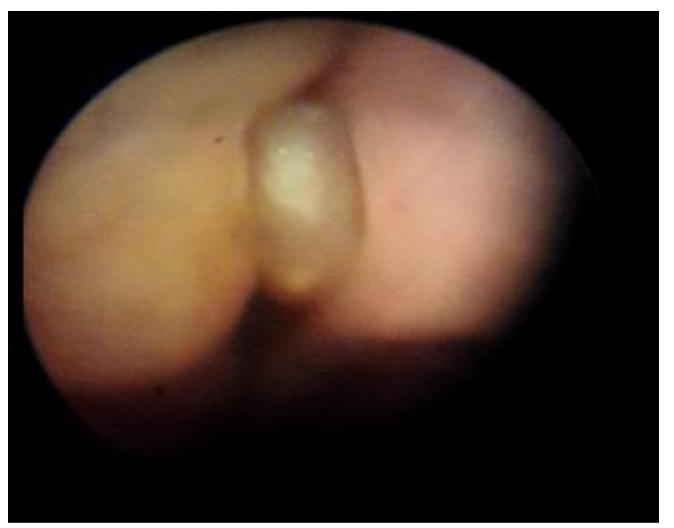


Figure 6: Polyposis biopsy: a) Low magnification: showed respiratory mucosa with perivascular infiltration of inflammatory cell; b) High magnification: demonstrated acute rhinitis ulcerated lesion with vasculitis extravascular eosinophilic infiltrates.

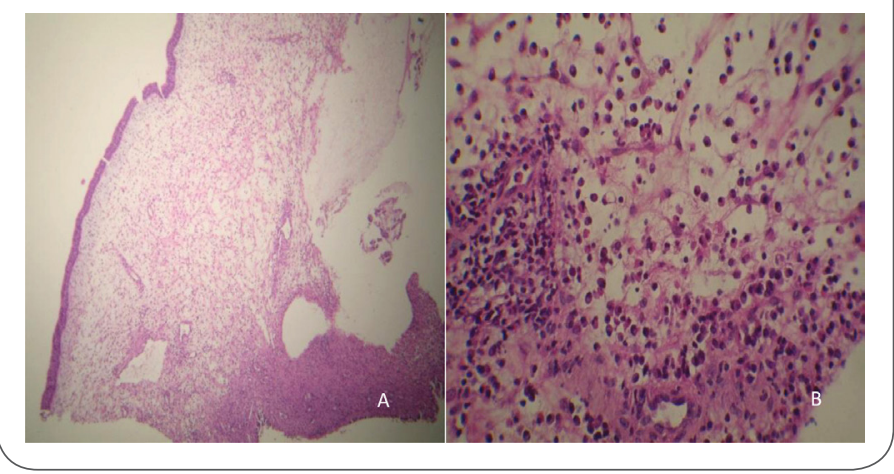

\section{Discussion}

According to the American College of Rheumatology, a patient can be diagnosed with CSS if at least four of the following six criteria of the traditional format classification are met, with a sensitivity of $85 \%$ and specificity of $99.7 \%$ : asthma, eosinophilia $>10 \%$ on a differential white blood cell count, mononeuropathy or polyneuropathy, nonfixed pulmonary infiltrates on roentgenography, paranasal sinus abnormalities or a biopsy containing blood vessels with extravascular eosinophils [4]. Our patient had four of these criteria: asthma; hypereosinophilia $>10 \%$, chronic sinusitis; and extravascular eosinophilic infiltrates (Figure 6).

We herein described a patient who presented with reccurent chest pain caused by coronary vasospasm. His symptoms of asthma, sinusitis, eosinophilia and allergic states, such as nasal polyps, led to the diagnosis of CSS. This is a rare case in which a CSS patient exhibited severe coronary artery spasms without a provocation test. Cardiac involvement is frequently observed in patients with CSS and is the leading cause of mortality. Cardiopathy is a common complication of CSS; myocarditis, congestive heart failure and valvular heart disease are usually observed [5]. Although coronary vasospasms are an unusual clinical manifestation of CSS $[6,7]$, the present case suggests that coronary vasospasms should be suspected in cases of CSS.

In one CSS patient described in the litterature with recurrent episodes of unstable angina pectoris, coronary angiography showed spontaneous coronary artery spasms [2]. In that patient, coronary angiography showed healthy coronary arteries, with spontaneous Vasospastic stenosis of the mid of the left anterior interventricular artery (LAD) and the left marginal artery. the intracoronary nitroglycerine injection showed their resolution (Figure 3 ). This is the second case in which a CSS patient exhibited severe coronary artery spasms without a provocation test.

Coronary artery vasospasm can causes myocardial infarction. Several studies demonstrate that severe spasm can lead to myocardial infarction. But This is the first case of CSS described in the literature who coronary vasospasm induced myocardial infarction [9].

Tansthoracic echocardiographie find severe left ventricular dysfunction with akinesia of the inferior well, the basal and the mid segment of the septum, the mid and apical segment of the inferolateral well, the basal of the anterior well and hypokinesia of the others wells, with severe alteration of global longitudinal strain $-9.5 \%$ (Figure 2).

Typical cardiac MRI findings in CSS include high signal intensity of myocardium with multifocal delayed enhancement, mainly subendocardial area, suggesting the multifocal myocarditis due to the small vessel vasculitis [10]. In the present case cardiac MRI find a systolic left ventricular dysfunction with transmural ischemic involvement who confirmed the myocardial infarction (Figure 4).

The major factors involved in the development of coronary vasospasms are endothelial dysfunction via abnormalities of nitric oxide (NO) 
synthase and its reduced bioavailability and hypercontractility of the vascular smooth muscle in spastic arteries that why eosinophilia may play an important role in coronary vasospasms and further studies are needed to evaluate the relationship between coronary vasospasms and eosinophilia. Vasodilators, such as calcium antagonists and nitrates, are effective in treating regular coronary vasospasms [11]. In contrast, coronary vasospasms in patients with eosinophilia are often resistant to vasodilators and may be responsive to immunosuppressive agents [12]. The present patient remained symptomatic under calcium antagonists, which lead us to initiate the combination therapy of glucocorticoids and cyclophosphamide with favourable clinical course.

The exact pathogenesis of CSS is unknown. Recent results suggest that eosinophil infiltration and ANCA-induced endothelial damage are likely the most important mechanisms in CSS [13]. The differences between ANCA-positive and ANCAnegative CSS cases were recently emphasized [13]. Similar to that observed in the present case, compared with ANCA-positive patients, ANCAnegative patients tend to have a high incidence of heart involvement [13]. Activated eosinophils may be the major pathogenic factor in ANCA-negative CSS cases.

In the present case symptoms of angina and coronary artery spasms disappeared following the administration of immunosupresseur. This suggests that her coronary vasospasms were provoked by hypersensitivity or an elusive autoimmune mechanism based on eosinophil infiltration.

\section{Conclusion}

Churg-Strauss syndrome (CSS) is a rare illness with clinical findings characterized by asthma, eosinophilia, and vasculitis affecting medium and small-sized arteries and veins in a variety of organs. The present case demonstrated that coronary artery vasos- pasm may develop as an unusual form of cardiac involvement in the course of CSS and can causes myocardial infarction. The reported patients were refractory to vasodilator treatment but successfully treated with immunosuppressive therapy. A differential white blood cell count should be obtained in patients with severe coronary spasm who are refractory to treatment with vasodilators to evaluate the possibility of CSS or other allergic states as potential differential diagnoses.

\section{References}

1. Noth I, Strek ME, Leff AR. Churg-Strauss syndrome. Lancet 361: 587-594, 2003.

2. Petrakopoulou P, Franz WM, Boekstegers P, Weis M. Vasospastic angina pectoris associated with Churg-Strauss syndrome. Nat Clin Pract Cardiovasc Med 2: 484-489, 2005.

3. Kozak M, Gill EA, Green LS. The Churg-Strauss syndrome. A case report with angiographically documented coronary involvement and a review of the literature. Chest 107: 578-580, 1995.

4. Masi AT, Hunder GG, Lie JT, Michel BA, Bloch DA, Arend WP, et al. The American College of Rheumatology 1990 criteria for the classification of Churg-Strauss syndrome (allergic granulomatosis and angiitis). Arthritis Rheum 33: 1094-1100, 1990.

5. D'Amico G, Nai Fovino L, Cucchini U, Cacciavillani L, lliceto S, Buja P. Non-culprit coronary vasospasm in a woman affected by Churg-Strauss syndrome presenting with ST-elevation myocardial infarction. Int J Cardiol. 2014 Nov 15;177(1): e10-2.

6. Wong CW, Luis S, Zeng I, Stewart RA. Eosinophilia and coronary artery vasospasm. Heart Lung Circ 17: 488-496, 2008.

7. Suzuki N, Arai Y, Miyamoto $Y$, Isokane N, Fukushima N, Sano $\mathrm{Y}$. Acute myocardial injury and repeated angina pectoris-like attacks in a young patient with Churg-Strauss syndrome. Nihon Kyobu Shikkan Gakkai Zasshi. 1991; 29(12): 1630-1637.

8. Tanaka M, Mise N, Kurita N, Suzuki T, Hara K, Fujii A, et al. Case of Churg-Strauss Syndrome With Necrotizing Crescentic Glomerulonephritis Accompanied by Acute Coronary Syndrome Due to Vasospasm. Am J Kidney Dis. 2010 Aug;56(2): e5-9.

9. Maseri A, L'Abbate A, Baroldi G. et al. Coronary vasospasm as a possible cause of myocardial infarction. A conclusion derived from the study of "preinfarction" angina. N Engl J Med. 1978;299: 1271-1277. 
10. Sauvetre G, Fares J, Caudron J, Dacher JN, Girszyn N, Daragon $A$, et al. usefuless of magnetic resonance imaging in churgstrauss syndrome related cardiac involvement. A case series of three patients and literature review. La revue de médecine interne 31 (2010) 600-605.

11. Stern S, Bayes de Luna A. Coronary artery spasm: a 2009 update. Circulation 119: 2531-2534, 2009.

12. Owen WF Jr, Soberman RJ, Yoshimoto T, Sheffer AL, Lewis RA, Austen KF. Synthesis and release of leukotriene C4 by human eosinophils. J Immunol 138: 532-538, 1987

13. Suzuki Y, Nishiyama O, Sakai T, Niiyama M, Itoh T, Nakamura M. Acute Coronary Syndrome Caused by Coronary Vasospasms Associated with Churg-Strauss Syndrome: Effects of Betamethasone Therapy. Intern Med 53: 717-720, 2014.

Publish in International Archives of Medicine

International Archives of Medicine is an open access journal publishing articles encompassing all aspects of medical science and clinical practice. IAM is considered a megajournal with independent sections on all areas of medicine. IAM is a really international journal with authors and board members from all around the world. The journal is widely indexed and classified Q2 in category Medicine. 